Cómo citar este artículo: Trujillo Quintero, H. F., Losada Cubillos, J. J. \& Rodríguez Zambrano, H. (2017, julio-diciembre) Amazoniacolombiana, petróleo y conflictos socio-ambientales. Rev. Cient. Gen. José María Córdova, 15(20), 209-223. DOI: http:// dx.doi.org/10.21830/19006586.181

\title{
Amazonia colombiana, petróleo y conflictos socioambientales*
}

DOI: http://dx.doi.org/10.21830/19006586.181

Recibido: 10 de septiembre de 2016 - Aceptado: 5 de junio de 2017

The Colombian Amazon: Oil Exploitation and Socio-Environmental Conflicts

L'Amazonie colombienne: l'exploitation pétrolière et des conflits socio-environnementaux

Amazônia Colombiana: petróleo e conflitos sócio-ambientais

Hernán Felipe Trujillo Quintero ${ }^{a}$
Jhon Jairo Losada Cubillos ${ }^{b}$
Hernando Rodríguez Zambrano ${ }^{c}$

* Documento elaborado dentro del proyecto de investigación "Extractivismo, conflicto armado y tensiones del Desarrollo en la Amazonia Colombiana desde un escenario de posconflicto: Lectura desde la Ecología Política y la Decolonialidad”, en el marco de la convocatoria 011 del Sistema Bonaventuriano de Investigaciones. Presenta resultados preliminares.

a Universidad de San Buenaventura, Bogotá, Colombia. <htrujillo@usbbog.edu.co.>.

b Universidad de San Buenaventura, Bogotá, Colombia. <jlosada@usbbog.edu.co.> .

c Universidad de San Buenaventura, Bogotá, Colombia. <hrodriguez@usbbog.edu.co.>. 
Resumen. La Amazonia colombiana presenta la mayor área de conservación ecológica del país. Es una zona donde interactúan factores bióticos — especies humanas y no humanas - y elementos abióticos agua, minerales, hidrocarburos-. Teniendo en cuenta que la industria petrolera tiene un interés especial sobre este territorio, con cerca de 23,7 millones de hectáreas, en este artículo se abordan los principales impactos socioambientales de la actividad petrolera en este territorio colombiano y las tensiones que genera con las comunidades humanas que lo habitan. Además, se plantea un criterio de sustituibilidad para garantizar la vida y la cultura en este territorio.

Palabras clave: Amazonia colombiana; conflictos socioambientales; petróleo.

Abstract. The Colombian Amazon has the larger ecological conservation area in the country. It is a region where there is an interaction between biotic factors - human and non-human species - and abiotic factors - water, fuel oil, minerals. Considering that the oil industry has a special interest on the Amazon territory, with about 58,5 million of hectares, this paper deals with the question concerning the main environmental impacts of the oil industry on the region, and the social tension which that causes with the human communities that inhabit the Amazon. Thus, a substitution criterion is proposed in order to safeguard the life and the culture in this part of the Colombian territory.

Keywords: Colombian Amazon; oil industry; socio-environmental impacts.

Résumé. L'Amazonie colombienne a la plus grande surface de conservation écologique du pays. C'est une région où interagissent des facteurs biotiques — des espèces humaines et non humaines — et des facteurs abiotiques — de l'eau, des minéraux, des hydrocarbures. Considérant que l'industrie pétrolière a un intérêt particulier sur ce territoire, avec près de 23,7 millions d'hectares, cet article aborde la question des principaux impacts socio-environnementales de l'activité pétrolière dans la région, et les tensions sociales que cela entraîne avec les communautés humaines qui habitent l'Amazonie. Il se pose donc un critère de substituabilité pour garantir la vie et la culture dans cette partie du territoire colombien.

Mots-clés: Amazonie colombienne; conflits socio-environnementaux; industrie pétrolière.

Resumo. A Amazônia colombiana tem a maior área de conservação ecológica do país. É uma região onde interagem elementos bióticos humanos e não humanos, e abióticos como água, minerais e hidrocarbonetos. Tendo em conta que a indústria do petróleo tem um interesse especial neste território, com cerca de 23,7 milhôes de hectares, neste artigo são abordados os principais impactos sócio-ambientais da atividade petrolífera da região, e as tensôes geradas com as comunidades humanas que habitam a Amazônia. Assim se propóes um critério de substituição para garantir a vida e cultura nesta parte do território colombiano.

Palavras chave: Amazônia colombiana; conflitos sócio-ambientais; indústria do petróleo.

\section{Introducción}

Colombia mantiene una dependencia ecológica con la Amazonia, que ocupa un poco más del $40 \%$ del territorio continental, aunque buena parte de quienes habitan el país no la conocen. Reconocida por el Fondo Mundial para la Conservación como uno de los ocho ecosistemas estratégicos para la humanidad (Sierra, 2003), allí confluyen biomas que se localizan a alturas de entre los 50 y 1.100 metros sobre el nivel medio del mar, donde el clima es de tipo cálido tropical 
y húmedo, y ligeramente estacional, con ecosistemas de bosques húmedos selváticos, sabanas inundables y sabanas altas.

Sobre el mismo territorio también coexisten una serie de tensiones entre el ser humano y el entorno que afectan las condiciones de vida de los habitantes del gran bosque húmedo colombiano. Estas tensiones empiezan a ser evidentes en los últimos años debido a los cambios que generan las actividades extractivas allí desarrolladas sobre los recursos que garantizan la satisfacción de necesidades de los habitantes rurales, sin mencionar los efectos adversos sobre otras comunidades, otras regiones u otros continentes. Así, además de los servicios de contemplación que los ecosistemas amazónicos pueden prestar, la importancia de la región a nivel mundial se explica por su capacidad para regular el clima regional, por ser un enorme albergue de biodiversidad, por contener la mayor cuenca hidrográfica del mundo, por ser uno de los mayores stocks de carbono del planeta y por contener una multiplicidad de culturas y lenguas (Comisión Económica para América Latina y el Caribe [CEPAL] \& Patrimonio Natural, 2013).

Los impactos asociados a las fases petroleras generan resistencias dentro de las comunidades locales receptoras, las cuales son cada vez más conscientes de los frágiles equilibrios que sostienen la vida (humana y no humana) en sus territorios y no encuentran un beneficio palpable en las actividades que modifican su entorno y, en consecuencia, afectan su bienestar. En este mismo sentido, la sociedad civil empieza a llamar la atención de estos conflictos ecológicos en la medida en que se hace evidente un régimen de distribución inequitativa de recursos bióticos y abióticos que afectan el bienestar de la civilización humana, y que el mercado no internaliza dentro de la información que se entrega a los mercados: el precio.

La economía ecológica y la ecología política, en los últimos años, han abordado dichas problemáticas, también denominadas como "conflictos socioambientales", "conflictos ecológicos" o "conflictos ecológico-distributivos" (Martínez-Alier, 2006). Dicho enfoque conceptual exige la revisión de los siguientes elementos: i) presencia de comunidades humanas en conflicto; ii) uso o agotamiento de recursos bióticos y/o abióticos como fuente del conflicto, iii) dos actores: algunos con un alto interés en la extracción y/o explotación del recurso objeto del conflicto, y otros con grandes afectaciones y/o con oposición a la extracción y/o explotación del recurso, iv) un territorio plenamente identificado, donde se desarrolla el conflicto, y $v$ ) relaciones de poder subordinadas entre los actores en oposición, bien sea por el poder legal, las relaciones de capital o el tamaño de los actores.

Si bien existe una densidad poblacional baja, los conflictos sobre el uso y abuso de los recursos naturales comienzan a marcar una tensión importante en las comunidades humanas que afecta la estabilidad socioecológica del territorio. Con base en lo anterior, se plantea como pregunta de investigación la siguiente: ¿Cuál es el impacto que genera la exacerbación de los conflictos socioambientales derivados de los intereses geográficos de la industria petrolera sobre la región de la Amazonia en Colombia?

Como respuesta a la pregunta de investigación y a partir de fuentes documentales, el propósito de este documento es identificar cuál es el impacto que genera la exacerbación de los conflictos socioambientales derivados de los intereses geográficos de la industria petrolera sobre la región de la Amazonia en Colombia. 


\section{Marco de referencia}

El funcionamiento de los socioecosistemas depende, necesariamente, de los equilibrios existentes en los ecosistemas alrededor de los flujos de materia y energía, o del metabolismo social (Martínez-Alier, 2006). Su conservación, sin embargo, enfrenta una tensión con la extracción de hidrocarburos presentes en el subsuelo de la región.

Luego de la creación de la Agencia Nacional de Hidrocarburos (ANH) en el ańo 2003, se incrementaron las asignaciones de bloques petroleros en los territorios colombianos con potencial de hidrocarburos. Asimismo, este hecho, aunado a la confianza inversionista en los últimos ańos gracias a la estabilidad macroeconómica y a la política de seguridad democrática, motivó la llegada de inversión extranjera directa en el sector minero-energético (Asociación Colombiana de Petróleo [ACP], 2010; Trujillo, 2008; Vélez \& Rocha, 2006; Zerda, 2011). En la Amazonia, la actividad petrolera inició en la década del setenta en los territorios del piedemonte de la cordillera Oriental, en el departamento de Putumayo.

Si bien los territorios concesionados para la explotación petrolera se concentraron en el piedemonte de la codillera Oriental, en los últimos dos años se han empezado a entregar bloques para exploración, explotación y producción en buena parte de las altiplanicies de la Amazonia (Finer \& Jenkins, 2008), donde se concentran los ecosistemas mejor conservados del país y con mayor importancia global.

El panorama del sector en estos territorios se presenta así: los departamentos de Caquetá y Putumayo representan más del $90 \%$ del total de proyectos exploratorios o de producción de hidrocarburos en la Amazonia, y en los departamentos del Meta, Casanare y Arauca se concentra el $97 \%$ del total de proyectos exploratorios en la Orinoquia (Cepal \& Patrimonio Natural, 2013). Así, cerca del 50\% del total de la actividad exploratoria en Colombia se desarrolla en la región Orinoco-Amazónica, y se espera una mayor participación en los próximos años, como lo confirma la ronda de proponentes del 2010, donde se escogieron las firmas que contratan con la ANH para realizar la exploración, explotación y producción petrolera—open round 2010_. En esa oportunidad se asignaron nuevos bloques en zonas cercanas a parques naturales, reservas forestales y resguardos indígenas, y según las proyecciones de la $\mathrm{ANH}$ y el mapa de tierras (Agencia Nacional de Hidrocarburos [ANH], 2010), para las próximas rondas se seguirán realizando nuevas asignaciones en territorios de la Amazonia que no se encuentran en parques naturales (Cepal \& Patrimonio Natural, 2013).

La pertinencia de la entrega de bloques petroleros en la Amazonia colombiana debe repasar los impactos que esta actividad económica extractiva tiene en relación con el ambiente y con las comunidades que allí viven, por lo cual se entrará a evaluarlos para el caso colombiano. Los límites físicos de los recursos naturales no renovables están disminuyendo sus reservas considerablemente debido a su sobreexplotación desde finales del siglo XIX. Este hecho y los altos precios del crudo en el periodo 2001-2014 incrementaron la actividad exploratoria en zonas donde su entrada es difícil o el petróleo es de menor calidad, como es el caso de la Amazonia, en donde se presentan estas dos situaciones, lo cual aumenta los costos energéticos y la cantidad de materiales removidos por unidad de recurso obtenido, y con ello, los impactos ambientales. Martínez-Alier y Joan (2000) señalan que los límites del recurso no renovable no se encuentran solamente en su 
agotamiento físico ni en las crecientes dificultades de su extracción, sino también en los impactos ambientales que tiene dicho recurso, con lo cual recuerda el doble papel que tiene la naturaleza como proveedora de recursos y receptora de residuos.

De manera general, las afectaciones a la biodiversidad de los ecosistemas tropicales en la Amazonia se presentan debido al incremento de la contaminación y de la deforestación (Avellaneda, 2009, 2004; Azqueta \& Delacámara, 2008; Bravo, 2007; Dureau \& Flórez, 1999; Dureau \& Goueset, 2001; Finer \& Jenkins, 2008; Fontaine, 2004; Trujillo, 2009). Los contaminantes pueden ser de distinta naturaleza: i) químicos, incluidos el petróleo y sus componentes, que ingresan al ambiente a través de las distintas prácticas operacionales, y los usados para facilitar la extracción petrolera; ii) sonoros, por las detonaciones que tienen lugar en la prospección sísmica y por el funcionamiento de la maquinaria petrolera; y iii) lumínicos, generada en la quema de gas y la utilización de energía para las operaciones de la planta y campamentos. Cada uno de estos contaminantes produce distintos tipos de impactos en la biodiversidad y en el ambiente, los cuales han sido retratados por la literatura científica (Azqueta \& Delacámara, 2008; Bravo, 2007).

Asimismo, la actividad petrolera genera deforestación en zonas densamente boscosas para instalar la infraestructura, construir los campamentos y para abrir y empalizar las carreteras, que a su vez constituyen una puerta abierta a la colonización y, a la postre, a una mayor deforestación. En este sentido, además de los espacios estrictamente deforestados, hay un efecto de borde que hace que la extensión alterada sea mucho mayor, lo cual provoca serios impactos en la fauna de los ecosistemas cercanos, sobre todo en animales mayores y en las aves, que huyen del lugar y, en consecuencia, afectan la alimentación y la salud de los pobladores, en su mayoría indígenas que viven de la caza (Bravo, 2007; Finer \& Jenkins, 2008).

Igualmente, las actividades de extracción petrolera impactan negativamente en las estructuras comunitarias de quienes habitan la Amazonia (Dureau \& Flórez, 1999; Dureau \& Goueset, 2001; Fontaine; 2004). Se ha logrado demostrar que generan procesos migratorios que modifican la estructura social y cultural de comunidades nativas (Bustamante \& Jarrín, 2004), no generan mayores coberturas de servicios públicos y no mejoran los indicadores de Necesidades Básicas Insatisfechas (NBI) de las poblaciones que habitan el área de influencia de los proyectos petroleros (Dureau \& Flórez, 1999; Dureau \&Goueset, 2001).

Para Colombia, se tienen evidencias científicas de los impactos de las actividades petroleras en los campos sociales y ambientales. En el tema social, Dureau y Flórez (1999) señalan que el descubrimiento y explotación del pozo petrolero Cusiana, ubicado en el departamento del Casanare, generó unas expectativas muy altas en los habitantes colombianos, lo cual aceleró un proceso de migración de personas hacia estos territorios en busca de nuevas oportunidades. Este hecho agudizó los conflictos sociales debido a que las nuevas ciudades - Yopal y Aguazul— empeoraron su seguridad por la llegada de actores armados ilegales que pretendían capturar las rentas petroleras. Además, en cuanto al mercado laboral, los autores indican que la excesiva oferta de mano de obra no calificada en la región llevó a las petroleras a rotar el trabajo cada cierto tiempo, generalmente cada tres o cuatro meses, con el fin de disminuir las demandas sociales y los descontentos que ello produce. Esta situación, sin embargo, generó inestabilidad y temporalidad en el trabajo petrolero, traducido en mayor incertidumbre para los habitantes de los municipios del Casanare. 
Por su parte, Hernández (2004) encontró que los indicadores sociales y de prestación de servicios públicos para departamentos como el Meta, el mayor productor de crudo en el país, se encuentran por debajo del promedio nacional. Así, los servicios públicos básicos como acueducto, alcantarillado, energía eléctrica, educación y salud no evidencian mejoras significativas pese a las orientaciones dadas por la normatividad que estipula la destinación de las regalías.

Por último, Bustamante y Jarrín (2004) describen que la situación de las zonas de la Amazonia ecuatorianas donde hay actividad petrolera tiene dos características. La primera tiene que ver con un importante déficit en todo lo que implica infraestructura, equipamiento y calidad de la vivienda, así como una apreciable desventaja en indicadores referentes a educación, así como una situación más comparable a la media en lo relativo a las variables de pobreza e indicadores de salud. El análisis de correlaciones entre la actividad petrolera y los indicadores sociales concluye que la presencia de la actividad petrolera no demuestra que genera bienestar o malestar a las poblaciones de la región amazónica.

Para cerrar con los impactos sociales, debe señalarse que, si se revisan con atención los registros de bienestar para los habitantes de cada uno de los municipios de la región amazónica en Colombia, se halla un preocupante panorama socioeconómico. El NBI ${ }^{1}$ se encuentra por debajo del promedio nacional, y en la zona rural alcanza el $72 \%$. El Índice de Condiciones de Vida $(\mathrm{ICV})^{2}$ evidencia grandes desigualdades entre departamentos, siendo Caquetá el de mejor condición, y Guainía, Vaupés y Amazonas los de peores condiciones de vida. El Índice de Desarrollo Humano (IDH) se encuentra por debajo del promedio nacional en todas las entidades territoriales que conforman la Amazonia. El déficit de vivienda da cuenta de grandes brechas entre lo urbano y lo rural, aunque con rezagos en comparación con la cifra nacional. Las cifras de educación, salud y saneamiento básico también presentan un rezago en comparación con la media nacional, y la prestación de servicios públicos tienen grandes deficiencias en el servicio de electricidad, acueducto y alcantarillado (Trujillo, 2016).

En el tema de hidrocarburos y su impacto en el ambiente, Avellaneda (2009) describe los impactos ambientales de la actividad petrolera en los parques nacionales naturales en Colombia que se encuentran cercanos a su área de influencia. Argumenta que la actividad extractiva, en sus diferentes etapas, ocasiona contaminación y cambios en el uso del suelo, remoción de materiales para la construcción de vías e instalaciones y pozos, contaminación de aguas superficiales y subterráneas, y modificaciones bióticas sobre hábitats naturales. Específicamente para la Amazonia y la Orinoquia, señala que los impactos de la entrada de empresas de hidrocarburos ocasionaron remoción de cobertura vegetal y construcción de trochas de penetración, entre las que se pueden contar varios parques nacionales naturales y zonas de reserva forestal. Asimismo, señala que uno de los factores que en la década del setenta facilitaron la penetración de los colonos a la Reserva de La Macarena fue precisamente la trocha abierta durante las labores de prospección sísmica de hi-

1 El índice NBI toma valores de 0 a 1, siendo 0 la situación en donde no hay carencia de necesidades, y 1 donde hay carencia de todas las necesidades básicas. Para revisar la metodología del NBI, se recomienda revisar el siguiente documento: Feres \& Mancero (2001).

2 El ICV vincula los resultados de encuestas subjetivas de satisfacción con la vida realizadas a las familias con los factores objetivos determinantes de calidad de vida. Va de 1 a 10, siendo 10 la mejor situación para garantizar una adecuada calidad de vida, y 1 la peor situación para garantizar una adecuada calidad de vida. Para revisar la metodología del ICV, se recomienda consultar el documento en línea The Economist Intelligence Unit's quality-of-life index (s. f.). 
drocarburos. En algunos proyectos, tales como el Oleoducto Caño Limón-Coveñas, el Oleoducto El Porvenir-Velásquez y el Gasoducto Villavicencio-Bogotá, se intervinieron zonas de reserva forestal en la cordillera Oriental, con graves consecuencias sobre la estabilidad de las cuencas y la oferta ambiental de agua, además de que se puede considerar nula la recuperación de la cobertura vegetal removida, que suma varios cientos de hectáreas.

En otro de sus artículos, Avellaneda (2004) sostiene que la actividad petrolera en Colombia, luego de cien años de historia, ha contribuido a la transformación de los paisajes naturales, a la dinamización de la colonización, y a la formación de pueblos y regiones, con lo cual se ha llevado tras de sí a decenas de culturas indígenas e introducido patrones extractivos que han degradado el medio ambiente, aumentaron la corrupción política regional e incrementaron la violencia. Avellaneda pone como ejemplo los territorios del Magdalena Medio, los Llanos Orientales y la Amazonia, zonas con la mayor actividad petrolera y con los mayores conflictos sociopolíticos, mayor inseguridad ambiental y mayor pobreza. Específicamente en el tema ambiental, señala que la actividad petrolera es insostenible debido a la presencia de algunos factores en las zonas de extracción, relacionados con algunos impactos directos o indirectos al ambiente, sinérgicos o acumulativos, residuales o persistentes, generados durante el desarrollo de la actividad económica. En este sentido, afirma que la mala calidad ambiental de los proyectos de hidrocarburos, y la falta de integridad ecosistémica, manifiestan una desarmonía entre las actividades antrópicas y la naturaleza.

Así las cosas, la condición de vida de la población de la Amazonia y la vulneración de los ecosistemas presentes en el territorio contrastan con los grandes recursos que manejan las empresas transnacionales que ubican sus pozos petroleros allí. Esta contradicción, que no es otra distinta a la que busca dar respuesta el denominado desarrollo sostenible, cuyas bases se encuentran sentadas en el respeto por las generaciones futuras para que ellas también puedan garantizar la satisfacción de sus necesidades sin descuidar las necesidades presentes, lleva de fondo una disfonía en el campo ambiental y social, justamente porque lo que se discute debe poderse comparar. En este caso, la valoración económica se ha puesto de relieve para dirimir esta difícil situación, estimando cada una de ellas en términos de precios monetarios. La microeconomía ha simulado mercados para estimar los valores que tendrían en el mercado algunos bienes que, en esencia, se encuentran fuera de él, como el caso del ambiente.

\section{Postura de la ciencia económica frente a los bienes ambientales}

La ciencia económica se encarga de estudiar el comportamiento de los mercados, y en su racionalidad — especialmente la construida por la microeconomía—, asume que los procesos productivos y las transacciones solo se generan si benefician a todas las partes involucradas, lo cual da lugar a que los costos y beneficios derivados de esta relación afectan solamente a los participantes en la transacción (Edgeworth, 1891; Pigou, 1943; Samuelson, 1970; Trincado, 2008).

A pesar de este supuesto generalizado, los procesos económicos afectan a individuos, sociedades y ecosistemas que no están directamente involucrados en las transacciones, de tal suerte que 
las fallas de mercado resultan ser una distribución sesgada de los beneficios, donde el mercado no es capaz de reflejar el objeto de la transacción, ni mucho menos avisar su impacto en el bienestar social y en el ambiente (Leff, 1998, 2003; Martínez-Alier y Joan, 2000; Posada, 2007), de tal forma que se hace necesaria la intervención del Estado mediante soluciones de política (Pigou, 1943).

Como puede inferirse entonces, las conductas de los agentes económicos — familias, empresas y Estado- afectan la situación de bienestar de otros individuos humanos y otras especies vivas, sin que quien las produzca se vea afectado, a lo que se le conoce como una externalidad. Esto ocasiona que el beneficio o costo marginal social sea diferente al beneficio o costo marginal privado. El problema de las externalidades se encuentra en que dan incentivos erróneos desde el punto de vista social. Así, verbigracia, la explotación de un recurso natural no renovable debería reflejar el costo marginal del uso del recurso más el costo que ello ocasiona a los demás por su explotación, incluyendo los impactos ambientales y sociales, y los costos generados a las demás especies vivas.

En el caso de las externalidades positivas como la tecnología, estas se consumirán o producirán en una cantidad inferior a la socialmente óptima. Cosa distinta ocurre con las externalidades negativas como la contaminación y los impactos ambientales, donde se consumirán o producirán en una cantidad mayor a la socialmente óptima. Esta divergencia entre los beneficios y los costos que conduce a asignaciones ineficientes dan sustento a la intervención del Estado. En referencia a este tema, Coase (1981) estableció que cuando los derechos de propiedad están bien definidos y no existen costos de transacción, la presencia de externalidades no tendría que conducir a asignaciones ineficientes de recursos debido a que los agentes que son generadores y receptores de la externalidad estarían dispuestos a llegar a un acuerdo para internalizar el efecto externo.

Existen algunos bienes denominados de mérito (o necesidades preferentes) como la educación, la salud, la naturaleza y la ciencia, que por ser intrínsecamente deseables deberían ser provistos por el Estado para que se consumieran en cantidades mayores a las que los individuos elegirían por sí mismos. Lo mismo ocurre con las necesidades indeseables (como el crimen, la inseguridad, el alcoholismo, etc.), el mercado da respuesta contradictoria con respecto al óptimo social, por lo cual se hace necesaria la intervención del Estado mediante políticas públicas. Lo anterior se sustenta en la posibilidad que tiene el Estado de poseer más y contar con una mayor cantidad de información, así como tener mejor criterio que los individuos para tomar decisiones, evaluando los riesgos de mejor manera que el mercado (Musgrave, 1992).

De igual manera, el mercado no responde satisfactoriamente, o es lento en provocar efectos sobre los productores en situación de riesgo, incertidumbre o de elevada urgencia, como desastres naturales o convulsión social, de manera que mediante la implementación de políticas de Estado se pueden corregir estas situaciones. Por último, también se presentan fallas de mercado debido a la forma como se distribuye la riqueza, y la imposición y composición de los impuestos refleja la intervención del Estado (Dornbusch \& Fischer, 2008).

Con esta aproximación básica a las fallas de mercado y a la intervención del Estado, conviene examinar el comportamiento de los bienes ambientales y la sostenibilidad dentro de la economía, centrando nuestra atención en el sector de los hidrocarburos.

Desde la economía neoclásica, el principal problema de los bienes no mercadeables, como es el caso de los bienes ambientales y los recursos naturales, es que todos los flujos de bienes y servi- 
cios que proveen no tienen mercado — en términos de formación de precios comerciables-. Por esta razón, los bienes ambientales y los recursos naturales, en la mayoría de las veces, son tratados como bienes gratuitos debido a que aparentemente son propiedad de todos. La ausencia de los derechos de propiedad bien establecidos sobre los recursos imposibilita la asignación adecuada de un precio para el bien ambiental que haga que los recursos naturales y ambientales sean usados óptimamente, y como se mencionó en el acápite anterior, justifica la intervención del Estado (Menger, 2003).

La economía del bienestar trata de evaluar lo que sería una determinación óptima de la economía en términos de precios y cantidades de productos e insumos (Just, Hueth \& Schmitz, 1982). Para entender todo el problema ambiental desde el punto de vista económico, es importante tener presente algunos de los conceptos más importantes propuestos por la economía del bienestar. Un primer concepto, y quizás uno de los más importantes planteados por la economía del bienestar, es el excedente del consumidor. El excedente del consumidor (EC) trata de medir la ganancia o pérdida de bienestar experimentadas efectivamente por un individuo cuya situación se ve modificada por algún evento económico, como es el caso de un cambio en el precio o en la cantidad.

Con la agregación de los excedentes se plantea un problema de política pública, porque las medidas implantadas mejoran la posición de algunas personas y empeoran la posición de otras. Toda medida de política implicará beneficios para algunos y costos para otros. De esta manera, la aprobación de políticas que mejoren la posición de todos sin empeorar la posición de nadie sería una regla ideal pero imposible de alcanzar (Just, Hueth \& Schmitz, 1982). Una modificación de la anterior propuesta, que considera siempre la existencia de perdedores, es la compensación, que propone la comparación de los excedentes para los ganadores y para los perdedores (Menger, 2003).

A partir de esta comparación surgen tres situaciones: i) la suma de los excedentes del consumidor de los ganadores supera la suma de excedentes del consumidor de los perdedores. Si esto ocurre, los ganadores podrían transferir dinero a los perdedores de tal modo que los perdedores no queden en peor situación que antes. En la literatura sobre economía del bienestar esto es llamado una mejora en el sentido de Pareto. ii) La suma del excedente del consumidor de los ganadores sea menor que la suma del excedente del consumidor de los perdedores. Este caso es exactamente lo contrario al anterior, de darse esta situación se presentaría, entonces, un desmejoramiento en el sentido de Pareto. iii) La suma del excedente del consumidor de los ganadores sea exactamente igual a la suma del excedente del consumidor de los perdedores. Esta situación implica que una política no ofrece mejoramiento ni empeoramiento de la situación inicial. La economía de bienestar, entonces, asienta que los valores económicos se expresen en términos de disposición a pagar de los individuos (DAP), y la disposición a aceptar compensaciones (DAC) (Just, Hueth \& Schmitz, 1982).

La teoría del bienestar, sin embargo, conserva una estructura cerrada del flujo económico y no contempla las entradas al sistema de recursos naturales finitos, ni tampoco las salidas del sistema de residuos y energía disipada, lo cual no permite hacer una valoración del bienestar más allá de las preferencias humanas (Martínez-Alier \& Joan, 2000). Esto ha significado una economía de crecimiento infinito, que no tiene en cuenta la finitud de los recursos que son utilizados para la producción. 
La economía ecológica nace justamente como respuesta a la racionalidad económica del crecimiento sin límites, buscando una sostenibilidad ecológica de la economía. Esta incluye la economía ambiental neoclásica — que trabaja valores monetarios o crematísticos- y la trasciende al incluir también la evaluación física de los impactos ambientales de la actividad humana (Martínez-Alier \& Joan, 2000). Aquí la sustituibilidad entre los factores capital natural y capital manufactura, esencial para la valoración económica, no es posible, dado que hay bienes no renovables y condiciones ambientales que son insustituibles, y necesarias para la vida en la tierra. Las necesidades humanas, en la economía ecológica, se abordan como consumos necesarios de las personas que implican consumo de energía, y además, una repartición y apropiación equitativa de los recursos para garantizar la vida de todos los seres vivos, a partir de las necesidades y sus satisfactores (Leff, 1998; Max-Neff, 1994).

De cualquier manera, al margen de las discusiones de la tasa de extracción de hidrocarburos y la valoración de entornos ecosistémicos complejos a partir del bienestar económico, la actividad petrolera en la Amazonia avanza sin contemplar — valorar- las pérdidas generadas por la actividad en los factores bióticos y/o abióticos, así como las afectaciones a la estabilidad social de los territorios, lo cual empieza a incomodar a los actores que se relacionan allí. En los últimos años es notorio el malestar de las comunidades hacia actividades que modifican sus procesos de interrelación económica, cultural y social, y definitivamente la industria petrolera ha generado un malestar por lo que ha significado la intervención en el piedemonte putumayense y recientemente en San Vicente del Caguán y San José de Fragua, aunado a las malas experiencias de comunidades que habitan la Orinoquia colombiana.

La modificación de la vocación económica y los valores culturales (Fontaine, 2004) han generado resistencia en los habitantes rurales amazónicos, lo que se evidencia, por ejemplo, en las iniciativas populares de los municipios de San José de Fragua, Belén de los Andaquíes, Valparaíso, Paujil, Montañita, Puerto Rico y Doncello (Caquetá), donde los ciudadanos se han organizado para recolectar firmas con el propósito de consultar a la ciudadanía la conveniencia de desarrollar actividades petroleras en sus territorios a través de las denominadas Consultas Populares. Movimientos sociales como el Comité por la Defensa del Agua, la Vida y el Territorio, y el Observatorio Ambiental y de Paz de la Amazonia convocan continuamente movilizaciones en diferentes municipios del departamento del Caquetá, donde las autoridades municipales y departamentales se sintonizan con el malestar ciudadano.

De cualquier manera, al margen de la incomodidad que genera en la ciudadanía el volcamiento de un departamento eminentemente ganadero hacia la industria petrolera, el interés de la industria petrolera sigue vigente. Además, es claro que la Agencia Nacional de Hidrocarburos (ANH) tiene intereses sobre el territorio amazónico por su potencial en hidrocarburos.

Entre las diferentes categorías contractuales definidas por la $\mathrm{ANH}$, la industria petrolera tiene un interés sobre el 53\% del territorio amazónico (tabla 1). De manera específica, hay 396 mil hectáreas destinadas a actividades de explotación y producción, pero con un gran potencial en fase exploratoria en 10 millones de hectáreas. 
Tabla 1. Área reservada por la ANH para la actividad petrolera en la Amazonia

\begin{tabular}{lc}
\hline \multicolumn{1}{c}{ Categoría ANH } & Territorio (hectáreas) \\
\hline Área disponible & 6.852 .239 \\
Área reservada & 3.454 .902 \\
TEA & 3.086 .717 \\
Área exploración & 9.951 .717 \\
Área de producción & 392.979 \\
\hline Total área petrolera & $\mathbf{2 3 . 7 3 8 . 5 5 4}$ \\
\hline
\end{tabular}

Fuente: elaboración propia. Datos obtenidos de ANH (2017).

\section{Criterio de sustituibilidad como factor de conservación en la Amazonia}

Ahora bien, aquí conviene analizar los debates entre la naciente escuela de economía ecológica y los defensores de la microeconomía y de los modelos de crecimiento económico (Cuerdo, 2000), en relación con la imposibilidad de sustituir el factor capital-capital natural, capital manufacturado y la complementariedad cuando se trata de sustituir recursos naturales renovables y no renovables siguiendo la regla de sustituibilidad siempre que no se rebose la capacidad de carga de los ecosistemas.

En relación con los recursos naturales no renovables, el ser humano los ha explotado para luego ser dispersados como energía irreversible —entropía - sin pagar un costo de reposición o un costo de reproducción (Leff, 1998). Su utilización económica disminuye necesariamente el stock de reserva, pero es posible sustituirlo por recursos naturales renovables. La sustitución debe hacerse con un incremento de capital natural al menos en la misma proporción en que se agota el recurso no renovable.

Así las cosas, el incremento de actividades de explotación petrolera en la Amazonia, siguiendo el criterio de sustituibilidad entre recursos naturales no renovables y renovables, debe tener en cuenta el reemplazo del recurso natural que se agota por otro que sea renovable. Justamente, Daly (1998), hablando de la sustituibilidad entre estos factores, planteó una regla a la que denominó "cuasisostenibilidad", en la cual el uso del capital natural en el proceso productivo de un recurso natural no renovable exige una inversión compensatoria en un recurso renovable que lo sustituya.

Para la Amazonia colombiana, el criterio ambiental para aplicar la regla de cuasisostenibilidad planteada por Daly, puede definirse en función de la ampliación de los ecosistemas protegidos por el Sistema de Parques Nacionales Naturales (SPNN) presentes en la Amazonia, especialmente aquellos que constituyen el corredor ecológico entre los ecosistemas andinos y amazónicos.

Para no destruir el criterio de sustituibilidad entre el agotamiento de recursos naturales no renovables y la ampliación o reposición de recursos naturales renovables, el proceso de extracción 
de petróleo en áreas de piedemonte y algunas anexas al SPNN debe tener en cuenta que la tasa de extracción de los pozos petroleros debe ser siquiera igual a la tasa de reposición de bosques o a la tasa de ampliación de áreas protegidas. Actualmente el SPNN en la Amazonia cuenta con 9,5 millones de hectáreas (tabla 2).

Tabla 2. Áreas de parques nacionales naturales (PNN) en la Amazonia colombiana

\begin{tabular}{lcl}
\hline \multicolumn{1}{c}{ Nombre PNN } & $\begin{array}{c}\text { Extensión } \\
\text { (Hectáreas) }\end{array}$ & \multicolumn{1}{c}{ Departamento } \\
\hline Amacayacu & 293.500 & Amazonas \\
Alto Fragua Indi Wasi & 76.270 & Caquetá \\
Cahuinarí & 575.500 & Amazonas \\
La Macarena & 629.280 & Guaviare y Meta \\
La Paya & 422.000 & Putumayo \\
Río Puré & 999.880 & Amazonas \\
Serranía de Chiribiquete & 2.782 .353 & Caquetá y Guaviare \\
Serranía de los Churumbelos & 97.189 & Caquetá, Cauca, Huila y Putumayo. \\
Serranía (Cordillera) de los Picachos & 447.740 & Caquetá y Meta \\
Tinigua & 215.185 & Meta \\
Yaigoje Apaporis & 1.055 .740 & Amazonas y Vaupés \\
Nukak & 855.000 & Guaviare \\
Puinawai & 1.092 .500 & $15 \%$ del área del departamento del Guainía \\
Orito Ingi-Ande & 10.240 & Putumayo y Nariño \\
\hline & & \multicolumn{1}{c}{$\mathbf{9 . 5 5 2 . 3 7 7}$} \\
\hline
\end{tabular}

Fuente: elaboración propia. Datos obtenidos de la página web de Parques Nacionales Naturales (s. f.).

Así las cosas, por cada hectárea destinada a la conservación, hay 2,5 hectáreas proyectadas para la actividad petrolera. Desde la perspectiva económica arriba abordada, el criterio de sustituibilidad quedaría definido como la garantía de mantenimiento de los equilibrios bióticos - recursos renovables — con su interacción abiótica — recursos renovables y no renovables_-, priorizando la vocación del territorio desde dos perspectivas: vocación económica o vocación de conservación. Para lo anterior, habrá que tener en cuenta los territorios en perspectiva multidimensional, entendiendo que el interés sobre estos no solo es petrolero, y que tanto las entidades territoriales (municipios, departamentos) que ordenan el territorio según sus necesidades, como la vocación 
agroecológica del territorio que requiere de valores ecosistémicos subsisten —o se superponen-, por lo cual la compensación —o criterio de sustituibilidad - dependerá de consensos socioambientales que involucren a todos los actores que tienen interés sobre el territorio. De cualquier manera, con las actuales realidades del sector petrolero sobre la Amazonia colombiana, se requiere de un ajuste en el factor territorial que logre articular los elementos de la sustituibilidad con una mayor área destinada para garantizar el mantenimiento de factores bióticos — recursos renovables — sobre la sustracción de factores abióticos como los minerales — recursos no renovables-.

Solo de esta manera se pueden lograr los postulados de la sostenibilidad en la Amazonia colombiana, una coordinación entre la política de hidrocarburos y la política de conservación de ecosistemas que respete las áreas protegidas y las áreas de reserva forestal, y que la compensación de la extracción de hidrocarburos, junto con todos los impactos que de allí se derivan, sean subsanados con una mayor área conservada y protegida a perpetuidad, donde no se pueda realizar ningún proyecto económico.

\section{Conclusiones preliminares y recomendaciones para líneas de investigación}

La Amazonia colombiana enfrenta un incremento en la actividad petrolera que afecta considerablemente los biomas presentes en estos territorios. Resulta importante reconocer que la presencia de estas actividades, con grandes flujos de capital, no mejoran las condiciones socioeconómicas de las áreas de influencia de los proyectos petroleros, pero sí afecta los ecosistemas naturales del pulmón de la Tierra, lo cual plantea un reto en términos de desarrollo sostenible.

Como una propuesta para superar estas problemáticas, especialmente en materia ambiental, se plantea una coordinación entre la política de hidrocarburos y la política de conservación de ecosistemas que respete las áreas protegidas y las áreas de reserva forestal, y que la extracción de hidrocarburos, junto con todos los impactos que de allí se derivan, sea compensada con una mayor área conservada y protegida a perpetuidad, donde no se pueda realizar ningún proyecto económico y se dediquen estos territorios exclusivamente a la conservación.

Resultaría interesante en trabajos posteriores revisar los mecanismos institucionales de Colombia y otros países de la región para entrar con mayor detalle en una propuesta que desarrolle las ideas aquí expuestas. Asimismo, es importante establecer los corredores ambientales más idóneos para canalizar la propuesta, desde el campo biológico y ecológico, que permitan establecer los ecosistemas representativos que deben conservarse y articularse con la propuesta de ampliación de los parques nacionales en la Amazonia. Por último, pueden explorarse alternativas que permitan involucrar la problemática en la que se encuentra el factor social con el auge petrolero en la Amazonia, el otro gran componente del desarrollo sostenible. 


\section{Referencias}

1. Agencia Nacional de Hidrocarburos (ANH). (2010). Informe de bloques petroleros en Colombia. Bogotá D.C.: Agencia Nacional de Hidrocarburos.

2. Agencia Nacional de Hidrocarburos (ANH). (febrero, 2017). Mapa de tierras [página web]. Recuperado de http://www.anh.gov.co/Asignacion-de-areas/Paginas/ Mapa-de-tierras.aspx

3. Asociación Colombiana de Petróleo (ACP). (2010). Sector minero y petrolero en Colombia. Bogotá D.C.: Asociación Colombiana de Petróleo-CIMCO.

4. Avellaneda, A. (2009). Petróleo e impacto ambiental en Colombia. Revista de la Universidad Nacional (1944-1992), 6(24), 21-28.

5. Avellaneda, A. (2004). Petróleo, seguridad ambiental y exploración petrolera marina en Colombia. Revista Íconos, 21, 11-17.

6. Azqueta, D. \& Delacámara, D. (2008). El costo ecológico de la extracción de petróleo: una simulación. Revista de la CEPAL, 94, 59-73.

7. Bravo, E. (2007). Los impactos de la explotación petrolera en ecosistemas tropicales y la biodiversidad [documento en línea]. Recuperado de https://www.inredh.org/archivos/ documentos_ambiental/impactos_explotacion_petrolera_esp.pdf

8. Bustamante, T. \& Jarrín, M. (2004). Impactos sociales de la actividad petrolera en Ecuador: un análisis de los indicadores. Revista Íconos-FLASCO, 21, 19-34.

9. Coase, R. H. (1981). El problema del coste social. Hacienda Pública Española, 8, 245-274.

10. Comisión Económica para América Latina y el Caribe (Cepal) \& Patrimonio Natural. (2013). Amazonía posible y sostenible. Bogotá, D. C.: Cepal y Patrimonio Natural. Recuperado de http://www.cepal.org/colombia/noticias/ documentosdetrabajo/2/49742/Amazonia_posible_y_sostenible.pdf.

11. Daly, H. (1998). Crisis ecológica y sociedad. México: Varias.

12. Dornbusch, R. \& Fischer, S. (2008). Macroeconomía (7.a ed.). Madrid: McGraw Hill.

13. Dureau, F. \& Flórez, C. (1999). El sueño petrolero: movilidad social y dinámicas urbanas en Yopal, aguazul y Tauramena (Casanare). Revista Territorios, 119-138.

14. Dureau F. \& Gouëset V. (2001). El proceso migratorio y sus consecuencias sobre el poblamiento de las ciudades petroleras: realidades y representaciones colectivas en el caso de las ciudades de Casanare, Colombia. Scripta Nova, 94 (76), 10.
15. The Economist Intelligence Unit's quality-of-life index (s. f.) Recuperado de http://www.economist.com/media/pdf/ QUALITY_OF_LIFE.pdf

16. Edgeworth, E. (1891). On the Determinateness of Economic Equilibrium. Paper Relating to Political Economy, 2, 313-319.

17. Feres, J. C. \& Mancero, X. (2001). El método de las necesidades básicas insatisfechas (NBI) y sus aplicaciones en América Latina. Santiago de Chile: CEPAL.

18. Finer, M. \& Jenkins, C. (2008). Oil and Gas Projects in the Western Amazon: Threats to Wilderness, Biodiversity, and Indigenous Peoples. PlosOne, 2932.

19. Fontaine, G. (2004). Microconflictos ambientales y crisis de gobernabilidad en la Amazonia ecuatoriana. Revista Íconos- FLASCO, 21, 35-46.

20. Hernández, G. (2004). Impacto de las regalías petroleras en el departamento del Meta. En Ensayos sobre economía regional. Villavicencio: Banco de la República.

21. Just, R., Hueth, D. \& Schmitz, A. (1982), Applied Welfare Economics. New York: Prentice-Hall.

22. Lasso, F. (2004). La incidencia del gasto público social sobre la distribución del ingreso y la reducción de la pobreza. Bogotá, D. C.: Misión para el Diseño de una Estrategia para la Reducción de la Pobreza y la Desigualdad.

23. Leff, E. (1998). Saber ambiental: sustentabilidad, racionalidad, complejidad, poder. México: Siglo XXI.

24. Leff, E. (2003). Ecología y capital. Racionalidad ambiental, democracia participativa y desarrollo sustentable. México: Siglo XXI.

25. Martínez-Alier J. (2006). Los conflictos ecológico-distributivos y los indicadores de sustentabilidad. Polis, 13. Recuperado de http://polis.revues.org/5359.

26. Martínez-Alier, J. \& Joan, R. J. (2000). Economía ecológica y politica ambiental. México: Fondo de Cultura EconómicaPNUMA.

27. Max-Neef, M. (1994). Desarrollo a escala humana. Uruguay: Editorial Nordad-Comunidad.

28. Menger, C. (2003). Economía y bienestar económico. Barcelona: Ediciones Orbis.

29. Musgrave, M (1992). Hacienda pública. Teoría y aplicada. México D. F.: McGraw Hill.

30. Parques Nacionales Naturales. (s. f.). Sistema Único de Información Normativa [página web]. Recuperado de http://www.parquesnacionales.gov.co/portal/es/normativi$\mathrm{dad} /$ sistema-unico-de-informacion-normativa/

31. Pigou, A. (1943). The Classical Stationary State. Economic Journal, 53 (212), 343-351. 
32. Posada, L. H. (1997). Desarrollo económico sostenible, relaciones económicas internacionales y recursos minero-energéticos en Colombia [documento en línea]. Bogotá, D. C.: Universidad Nacional de Colombia. Recuperado de http://www.sernanp.gob.pe/documents/10181/88081/ Desarrollo+sostenible+economico.pdf/0e06a277-88f64596-9273-1ad4aac8f2d1

33. Samuelson, P. (1970). Economía. México: McGraw Hill.

34. Sierra, R. (2003). Dynamics and patterns of deforestation in the Western Amazon: The Napo Deforestation Front, 1986-1996. Applied Geography, 20 (1), 1-16.

35. Trincado, E. (2008). Economía de Desarrollo vs. Economía de Bienestar: distintas filosofías de vida. Revista de Economía Mundial, 18, 141-154.
36. Trujillo, H. F. (2008). Determinantes de la inversión extranjera directa en Colombia 1990-2007. Revista FENADECO, 84-96.

37. Trujillo, H. F. (2016). Relaciones económicas y conflictos socio-ambientales: el caso del territorio amazónico colombiano. Bogotá, D.C.: Ed. Bonaventuriana.

38. Vélez, M. \& Rocha, V. (2006). Inversión, confianza, mecanismos de promoción y protección a la inversión - contratos de estabilidad juridica. Bogotá, D.C.: Pontificia Universidad Javeriana.

39. Zerda, Á. (2011). Colombia: del Japón de Suramérica a la confianza inversionista: dos estrategias para un patrón de crecimiento reprimarizante con iniquidad. Bogotá, D.C.: Universidad Nacional de Colombia. Recuperado de http:// www.bdigital.unal.edu.co/3245/1/doczerda_fce_ee_24.pdf 